\title{
A Comparison of the Penetration and Permeation of Caffeine into and through Human Epidermis after Application in Various Vesicle Formulations
}

\author{
Eman Abd ${ }^{a, c}$ Michael S. Roberts ${ }^{a, b}$ Jeffrey E. Grice ${ }^{a}$ \\ a Therapeutics Research Centre, Translational Research Institute, School of Medicine, University of Queensland, \\ Brisbane, Qld., and ' School of Pharmacy and Medical Sciences, University of South Australia, Adelaide, S.A., \\ Australia; ${ }^{C}$ College of Health and Medical Technology, Baghdad, Iraq
}

\section{Key Words}

Vesicle systems · Stratum corneum · Hair follicles .

Encapsulation efficiency

\begin{abstract}
Background/Aims: A range of vesicles is now widely used to carry various solutes into and through the epidermis. These usually have the active solute encapsulated within and may be modified to confer flexibility and skin penetration enhancement. Here, we compared the ability of five different vesicle systems to deliver a model hydrophilic drug, caffeine, into and through excised human skin. Methods: In addition to lipids, the vesicle excipients included eucalyptol or oleic acid as penetration enhancers, and decyl polyglucoside as a non-ionic surfactant. Vesicle particle sizes ranged between 135 and $158 \mathrm{~nm}$, and caffeine encapsulation efficiencies were between 46 and $66 \%$. Caffeine penetration and permeation were measured using high-performance liquid chromatography. Results: We found that niosomes, which are liposomes containing a non-ionic surfactant, and transferosomes (ultraflexible vesicles) showed significantly greater penetration into the skin and permeation across the stratum corneum. Significant enhancement of caffeine penetration into hair follicles was found for transferosomes and those
\end{abstract}

liposomes containing oleic acid. Conclusions: We conclude that targeted delivery of the hydrophilic drug caffeine into the skin compartments can be modified using optimized vesicular formulations.

(c) 2015 S. Karger AG, Basel

\section{Introduction}

Topical drug delivery offers specific benefits over other administration routes for local action, but the barrier nature of the skin presents a major obstacle for many drugs delivered into and through it [1-3]. Chemical strategies to reduce the effect of the barrier include the use of penetration enhancers, such as oleic acid or octyl salicylate, and the use of more advanced colloidal systems such as microemulsions, solid lipid nanoparticles and nanostructured lipid carriers. Liposomes are hollow spheres comprised of phospholipid and an aqueous phase. Hydrophilic drugs may be inserted in the aqueous compartment, while lipophilic drugs can be sequestered in the phospholipid. Many excipients including lipids, surfactants and solvents have been added to improve the stability or other structural features of liposomes [4]. Cholesterol tends to make the liposome membrane less perme-

\section{KARGER}

E-Mail karger@karger.com

www.karger.com/spp (c) 2015 S. Karger AG, Base

1660-5527/15/0291-0024\$39.50/0
Michael S. Roberts

Therapeutics Research Centre, Translational Research Institute University of Queensland School of Medicine, Level 5

37 Kent Street, Woolloongabba, Brisbane, QLD 4102 (Australia)

E-Mail m.roberts@uq.edu.au 
able by filling up holes or disruptions [4]. Jacobs et al. [5] speculated that liposomes may either form a thin lipid film on the surface of the skin, so that water loss is retarded, or combine with intercellular skin lipids to form liquid-crystalline matrices in the uppermost skin layers. Strategies for lipid-based vesicle delivery systems have been reviewed by Lademann et al. [6] and by Williams [7], in his summary of the contribution by Brian Barry.

Various forms of liposomes exist. Transferosomes are defined as vesicular particles composed of phospholipids and surfactants such that the inner aqueous compartments are surrounded by a highly elastic and adaptable lipid bilayer [8]. They present a more flexible transport system with improved vesicular stability and the potential to be used with diverse active components [9]. Transferosomes are metastable, with an outer membrane that is ultraflexible and highly deformable [8], making them able to enter pores smaller than their size [10]. The flexibility is in part contributed to by their relatively high membrane hydrophilicity, which gives the membrane the ability to swell more than, for example, conventional liposomes. Like liposomes, transferosomes are unilamellar or multilamellar structures [11] and contain a surfactant as an edge activator $[12,13]$. Niosomes are unilamellar or multilamellar spheroidal structures containing a mixture of cholesterol and non-ionic surfactants such as alkyl ethers, esters or amides $[14,15]$. They have been assessed as an alternative drug delivery system compared to conventional liposomes.

Niosomes offer higher physical and chemical stability [16] and a great variety of surfactant choice [17]. It is claimed that for topical applications, niosomes can increase the time of drug residence in the stratum corneum and epidermis whilst decreasing drug systemic absorption [17]. After exposure of the skin to niosomes, another study observed structural changes in the lipid interstitial spaces of the stratum corneum and suggested that these changes may be responsible for the resultant enhanced penetration [18].

Skin appendages provide an alternative route for drug penetration through the skin. Pertinent to drug penetration via hair follicles, the follicular infundibulum provides an increase in the surface area, acting as a reservoir, and represents, at the lower end, an interruption of the stratum corneum barrier [19]. Some studies have focused on direct targeting of hair follicles such as with compounds for acne and alopecia therapies. Many attempts have been made to measure substances retained in the hair follicles after the topical administration of model compounds such as dyes [20] and nanoparticles [21].
However, little has been done with therapeutic agents and this subject requires further investigation [22].

This study aimed to investigate the feasibility of vesicle (liposome, transferosome and niosome) structure to improve the delivery of the hydrophilic drug caffeine into appendages, the stratum corneum and through human skin. Differential stripping [23] is a useful technique that allows measurement of the amount of solute retained in the stratum corneum by tape stripping and of the amount of follicular retention by follicular casting. In order to target a particular skin site after topical application, the formulation of caffeine was manipulated. Liposome, transferosome and niosome formulations were prepared by the thin-film hydration method using DLPC (dilauroylL- $\alpha$-phosphatidylcholine) and cholesterol (as lipid) and oleic acid, eucalyptol or decyl polyglucoside (DPGluc) as edge activators.

\section{Materials and Methods}

\section{Materials}

Caffeine (PubChem CID: 2519), oleic acid (PubChem CID: 445639), eucalyptol (PubChem CID: 2758), ethanol, cholesterol and DLPC (CAS No. 18194-25-7) were obtained from Sigma-Aldrich Pty. Ltd. (Sydney, N.S.W., Australia). DPGluc (sold as Oramix NS 10) was obtained from SEPPIC SA (France). All chemicals and solvents were of analytical grade.

\section{Vesicle Preparation}

Five different vesicles were prepared, with excipient mixtures as shown in table 1 . For all vesicles, the excipients were mixed in a rotary flask in the ratios shown, followed by glass beads $(4 \mathrm{~g})$ and chloroform $(3 \mathrm{ml})$. Once the lipids were dissolved, the solvent was gradually evaporated on a rotary evaporator at $30^{\circ} \mathrm{C}$ until a lipid film formed on the wall of the flask. The flask was set aside for $2 \mathrm{~h}$ to eliminate residual chloroform, after which the lipid film was stirred with $2 \mathrm{ml}$ of caffeine solution $\left(2 \%\right.$ in water) for $2 \mathrm{~h}$ at $30^{\circ} \mathrm{C}$. The liposome suspensions were extruded through a polycarbonate membrane with a pore size of $100 \mathrm{~nm}$ using the LiposoFast extruder (AVESTIN, Inc., Ottawa, Ont., Canada). Thirty-one cycles of extrusion were performed for each vesicle type.

\section{Vesicle Characterization}

The average particle diameter, polydispersity index and zeta potential of the vesicles were measured with a Zetasizer Nano ZS (Malvern, UK). Each sample was measured 3 times and the mean value was calculated.

\section{Caffeine Encapsulation Efficiency}

To separate the non-encapsulated drug, the vesicle suspensions were centrifuged at $14,000 \mathrm{rpm}$ for $30 \mathrm{~min}$ at room temperature. The amounts of caffeine in the supernatant and also in the clear solution were analysed by high-performance liquid chromatography (HPLC). Drug encapsulation efficiency (EE\%) was calculated as: $\mathrm{EE} \%=$ $100 \times$ mass of incorporated drug/total mass in vesicle preparation. 
Table 1. Liposome formulations showing excipient ratios and aqueous control composition

\begin{tabular}{lll}
\hline Name & Description & Composition \\
\hline $\mathrm{C}$ & Aqueous control solution & 100\% water \\
L & Conventional liposome & DLPC:cholesterol 35:7 \\
LEU & Conventional liposome + eucalyptol & DLPC:eucalyptol:cholesterol 35:7:7 \\
LOA & Conventional liposome + oleic acid & DLPC:oleic acid:cholesterol 35:7:7 \\
T & Modified liposome $\rightarrow$ 'transferosome' & DLPC:DPGluc:cholesterol 35:7:7 \\
$\mathrm{N}$ & Modified liposome $\rightarrow$ 'niosome' & DPGluc:cholesterol 35:735:7 \\
\hline
\end{tabular}

All formulations contained caffeine, $2 \% \mathrm{w} / \mathrm{v}$.
Table 2. Vesicle system characterization and encapsulation efficiency (means \pm SEM)

\begin{tabular}{lllrl}
\hline Formulation & Vesicle size, $\mathrm{nm}$ & $\begin{array}{l}\text { Polydispersity } \\
\text { index }\end{array}$ & Zeta potential & $\begin{array}{l}\text { Encapsulation } \\
\text { efficiency, \% }\end{array}$ \\
\hline L & $137.1 \pm 1.3$ & $0.06 \pm 0.03$ & $2.3 \pm 0.7$ & 48 \\
LEU & $142.3 \pm 2.3$ & $0.10 \pm 0.01$ & $-9.5 \pm 0.5$ & 66 \\
LOA & $128.4 \pm 1.9$ & $0.08 \pm 0.02$ & $-51.0 \pm 2.7$ & 50 \\
T & $158.3 \pm 4.0$ & $0.14 \pm 0.04$ & $-18.3 \pm 0.2$ & 47 \\
N & $135.2 \pm 2.3$ & $0.15 \pm 0.02$ & $-42.6 \pm 2.4$ & 46 \\
\hline
\end{tabular}

See table 1 for description of formulations.

\section{Caffeine Skin Permeation Studies}

Permeation studies using abdominal full-thickness human skin were performed in Franz diffusion cells with an effective diffusion area of $1.33 \mathrm{~cm}^{2}$ and a $3.4-\mathrm{ml}$ receptor chamber volume. The skin was provided by a female donor following elective surgery, with ethical approval granted by the University of Queensland Human Research Ethics Committee (Approval No. 2008001342). The skin was cut into discs and mounted in the Franz diffusion cells with the stratum corneum side facing upwards. The receptor contained PBS buffer ( $\mathrm{pH} 7.4$ ) at $35^{\circ} \mathrm{C}$. A volume of $160 \mu \mathrm{l}$ of the vesicle formulations and the control solution was applied to the surface of the skin and the donor chamber was covered in parafilm for the duration of the experiment to inhibit water evaporation. At various times over a 24 -hour period, $200 \mu \mathrm{l}$ of the receptor was withdrawn and replaced with the same amount of fresh PBS buffer. After the 24-hour sample was taken, the cells were dismantled and the skin in each cell was wiped with cotton buds to remove any remaining formulation. Up to 20 tape strips were then taken from each skin (D-Squame tapes; CuDerm Corp., Dallas, Tex., USA). The tapes were placed into separate vials and mixed overnight with $2 \mathrm{ml}$ of methanol to extract the caffeine removed from the stratum corneum by the tapes. Follicular casting was performed after tape stripping. One drop of superglue (LOCTITE ${ }^{\circledR}$; Henkel Australia) was placed on a microscope slide and pressed onto the surface of the stripped skin with light pressure for $15 \mathrm{~min}$. The slide was peeled carefully from the skin and the superglue containing caffeine sampled from the follicles was dissolved by rubbing 4 times with acetone-soaked cotton buds. The cotton buds were mixed overnight with $2 \mathrm{ml}$ of methanol. After follicular casting, the skin was chopped into small pieces, homogenized and mixed overnight with $2 \mathrm{ml}$ of methanol. Receptor fluid and extracts from tapes, casts and skin were analysed by HPLC.

\section{Sample Analysis}

Caffeine was analysed by a sensitive and rapid HPLC method. The HPLC system consisted of a Shimadzu SIL-20A HT, a CBM20A system controller, an SPD-20A detector, an LC-20AD pump and an auto injector. Isocratic separation was performed on a Phenomenex Luna C18 5- $\mu \mathrm{m}$ column $(150 \times 4.6 \mathrm{~mm})$, using 95\% water, $2 \%$ acetonitrile, $2 \%$ tetrahydrofuran and $0.5 \%$ acetic acid as the mobile phase, at a flow rate of $1 \mathrm{ml} / \mathrm{min}$. Caffeine was detected at $273 \mathrm{~nm}$.

\section{Data Analysis and Statistics}

The cumulative amount (micrograms/centimetre squared) penetrating per skin surface area was plotted against time (hours). The steady-state flux ( $\mathrm{J}_{s s}$; micrograms/centimetre squared/hour) was derived from the slope of the linear portion of the cumulative amount versus the time plot.

All data were analysed by one-way analysis of variance (ANOVA) with post hoc comparisons (Tukey) using GraphPad Prism 6 (GraphPad Software Inc., La Jolla, Calif., USA). $\mathrm{p}<0.05$ was considered to be significant.

\section{Results}

\section{Vesicle Characterization}

Particle size, polydispersity indices, zeta potentials and caffeine encapsulation efficiencies of all vesicles are presented in table 2 . As shown by the low polydispersity index values, all vesicles had a narrow size distribution. The high negative zeta potential values for both niosomes and 
Table 3. Permeation parameters following 24-hour application of caffeine from vesicle formulations and controls to full-thickness human skin (means $\pm \mathrm{SEM}, \mathrm{n}=4$ )

\begin{tabular}{|c|c|c|c|c|c|c|}
\hline Formulation & $\mathrm{J}_{\mathrm{SS}}, \mu \mathrm{g} / \mathrm{cm}^{2} / \mathrm{h}$ & $\begin{array}{l}\text { SC retention, } \\
\mu \mathrm{g} / \mathrm{cm}^{2}\end{array}$ & $\begin{array}{l}\text { Hair follicles, } \\
\mu \mathrm{g} / \mathrm{cm}^{2}\end{array}$ & $\begin{array}{l}\text { Skin retention, } \\
\mu \mathrm{g} / \mathrm{cm}^{2}\end{array}$ & $\begin{array}{l}\text { Receptor at } \\
24 \mathrm{~h}, \mu \mathrm{g} / \mathrm{cm}^{2}\end{array}$ & Total, $\mu \mathrm{g} / \mathrm{cm}^{2}$ \\
\hline $\mathrm{C}$ & $0.7 \pm 0.2$ & $17.3 \pm 4.2$ & $0.8 \pm 0.2$ & $48.4 \pm 7.8$ & $14.2 \pm 4.1$ & $80.7 \pm 12.4$ \\
\hline LEU & $1.8 \pm 0.7$ & $32.8 \pm 5.1$ & $1.6 \pm 0.4$ & $95.7 \pm 11.0$ & $35.8 \pm 15.8$ & $166.0 \pm 26.8$ \\
\hline LOA & $1.7 \pm 0.2$ & $39.4 \pm 6.4$ & $3.5 \pm 0.7$ & $93.5 \pm 7.8$ & $34.9 \pm 3.3$ & $147.9 \pm 30.7$ \\
\hline
\end{tabular}

See table 1 for description of formulations. SC = Stratum corneum. The total shown is the sum of retention in the stratum corneum, hair follicles, residual skin and receptor penetration. Significant differences (one-way ANOVA/Tukey): ${ }^{\text {a }}$ versus control solution and liposomes; ${ }^{b}$ versus control solution, liposomes, liposomes + eucalyptol and liposomes + oleic acid.

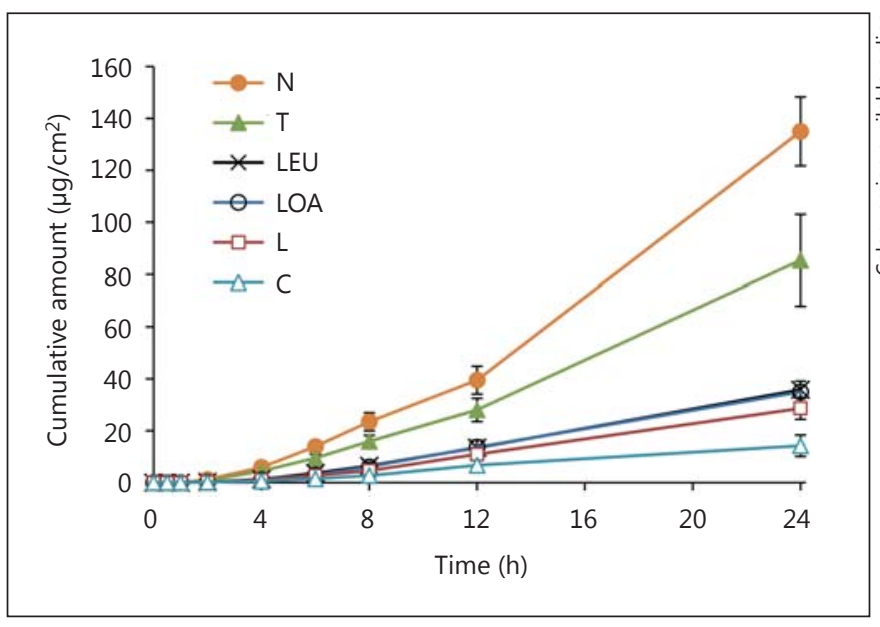

Fig. 1. Permeation profiles of caffeine through excised human skin from various vesicle systems and a control (means \pm SEM, $\mathrm{n}=4$ ). $\mathrm{N}=$ Niosomes; $\mathrm{T}=$ transferosomes; $\mathrm{LEU}=$ liposomes + eucalyptol; LOA = liposomes + oleic acid; $\mathrm{L}=$ liposomes; $\mathrm{C}=$ control solution.

the liposomes containing oleic acid indicate that these materials, in particular, would be stable in suspension. The encapsulation efficiencies were relatively similar across the five vesicle types.

\section{Permeation of Caffeine from Vesicles and Control}

The cumulative amounts of caffeine penetrating human full-thickness skin from each of the test formulations over $24 \mathrm{~h}$ are shown in figure 1, and overall steady-state fluxes calculated from these plots are shown in table 3.

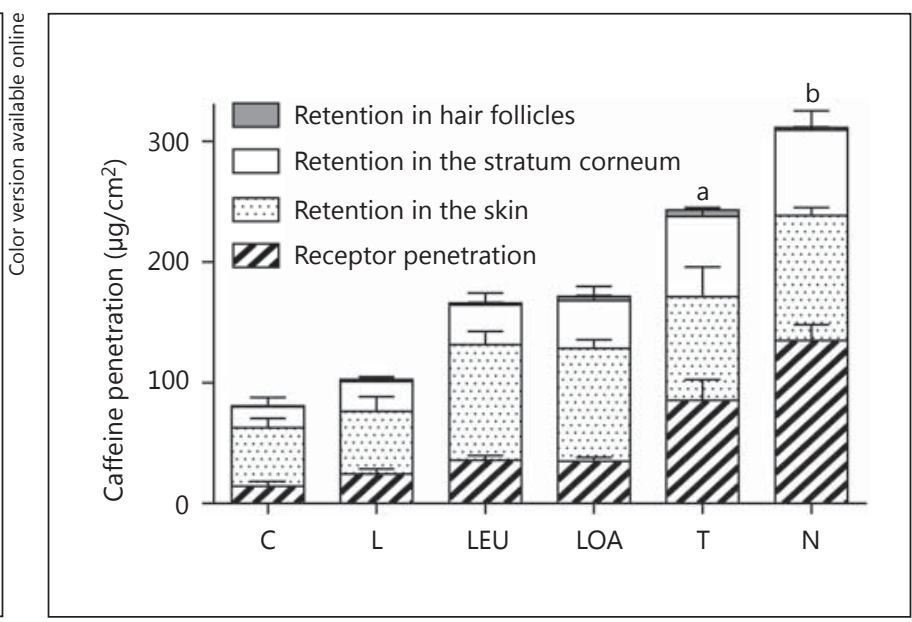

Fig. 2. Caffeine recovery from the skin surface, the stratum corneum, hair follicles, residual skin and receptor solution after application of vesicles and control for $24 \mathrm{~h}$ (mean \pm SEM, $\mathrm{n}=4$ ). For abbreviations, see legend to fig. $1 .{ }^{\text {a }}$ Amount in receptor at $24 \mathrm{~h}$; transfersomes $>$ control solution and liposomes. ${ }^{\mathrm{b}}$ Amount in receptor and total penetration at $24 \mathrm{~h}$; niosomes $>$ control solution, liposomes, liposomes + eucalyptol and liposomes + oleic acid (one-way ANOVA/Tukey).

Niosomes and transferosomes had significantly greater fluxes than other formulations, as indicated.

Other skin permeation parameters for caffeine from the six applications are illustrated in table 3 and figure 2 . Penetration of caffeine into the receptor was significantly enhanced from the niosomes compared to the aqueous control solution and the liposomes (liposomes, liposomes with eucalyptol and liposomes with oleic acid) and from the transferosomes compared to control and the basic liposomes. In addition, total skin penetration (stratum cor- 
neum, hair follicles, residual skin and receptor solution) was significantly enhanced from niosomes compared to control and the basic liposomes. Caffeine recovered from hair follicles after transferosome application was 50\% more abundant than with liposomes with oleic acid and more than twice that seen with all other formulations, although the differences did not reach statistical significance ( $p=0.10$, transferosomes vs. control). Similarly, the stratum corneum and skin retention were enhanced with the advanced formulations (niosomes, transferosomes and to a lesser extent, the liposomes containing penetration enhancers eucalyptol and oleic acid), but the differences did not reach statistical significance.

\section{Discussion}

The nanosize range of the vesicles used in this study $(128-158 \mathrm{~nm})$ makes them suitable for topical delivery. We [24] and others [25] have shown that by decreasing the vesicle size, the penetration of encapsulated drug into deeper skin layers can be enhanced. Further reductions in vesicle size could be achieved by extrusion through smaller membranes, particularly with ultraflexible materials such as transferosomes and SECosomes [24]. In addition to the narrow range of nanosizes in our series of vesicles, the individual vesicles were shown to be homogeneous, as indicated by the low polydispersity index values $(0.05-$ $0.15)$. However, given this homogeneity in particle size seen across the range of vesicle formulations, it is likely that factors other than particle size contributed to the observed differences in caffeine flux and retention in skin compartments (table 3; fig. 1,2). Regarding the zeta potential, liposomes with oleic acid and niosomes possessed a negative surface charge (table 2), while the dispersion of liposomes with eucalyptol, transferosomes and liposomes possessed a neutral charge, indicating that the incorporation of edge activators into liposomes had an important influence on their surface charge.

The encapsulation efficiencies seen here (46-66\%) compare well with $30 \%$ found by Budai et al. [26] for solid lipid nanocarriers based on soy or egg lecithin. Caffeine is soluble in water $(21.3 \mathrm{mg} / \mathrm{ml}$ at room temperature $)$ and in organic solvents has a low octanol/water partition coefficient $(\mathrm{P} ; \log \mathrm{P}=-0.07)$. Therefore, the caffeine must be able to enter the lipophilic and hydrophilic core of the vesicular structure [27]. The caffeine encapsulation efficiency was highest in the liposomes containing eucalyptol. This may be due to the relatively hydrophilic nature of eucalyptol, which results in low permeability of vesicles.
Before application to the skin, our caffeine liposome formulations were freshly prepared, characterized and stored briefly overnight at $4^{\circ} \mathrm{C}$. Several publications have explored the 'leakage' of similarly small hydrophilic molecules from vesicles upon storage. Fang et al. [11] (2001) found that the addition of cholesterol to the phospholipid DMPC (1,2-dimyristoyl-sn-glycero-3-phosphocholine) significantly reduced the leakage of enoxacin (log $\mathrm{P}=-0.2$, see caffeine, -0.07 ) from their vesicles over $48 \mathrm{~h}$ at $25^{\circ} \mathrm{C}$. All the vesicle formulations used in our study contained cholesterol. The authors also found that the presence of a non-ionic surfactant in the formulation tended to stabilize the vesicle. Balakrishnan et al. [14] (2009) showed that the loss of the small, relatively hydrophilic minoxidil $(\log \mathrm{P}=1.24)$ from niosomes containing a non-ionic surfactant and cholesterol was less than $5 \%$ after 14 days of storage at $4{ }^{\circ} \mathrm{C}$ - the same temperature used in this study. Ntimenou et al. [28] (2012) found a reduction in calcein encapsulation from conventional liposomes and transferosomes containing cholesterol and a surfactant, as well as 'invasomes', containing terpenes, of no more than $15 \%$ after $24 \mathrm{~h}$ at the relatively extreme temperature of $37^{\circ} \mathrm{C}$. The terpene mixtures performed slightly better than the other formulations. Taken together, these examples, covering a range of vesicles used in this study, indicate that $12 \mathrm{~h}$ of storage at $4^{\circ} \mathrm{C}$ would be unlikely to result in a significant loss of caffeine from the vesicles.

Optimized vesicular systems can exert different functions upon topical delivery [29]. They can improve drug deposition in the skin at the site of action, with the additional goal of decreasing systemic absorption and thus minimizing adverse effects [30]. They may also provide targeted delivery to skin appendages. In an attempt to find a vesicular formulation for topical delivery of caffeine, vesicular systems were formulated using oleic acid, eucalyptol and DPGluc as edge activators. Oleic acid and eucalyptol are well-known penetration enhancers [31]. DPGluc is a low-irritant, non-ionic surfactant that is commonly used in pharmaceutical and cosmetic formulations and can form vesicles including niosomes in combination with appropriate amounts of cholesterol [17].

In this work, all formulations were able to achieve sustained delivery of caffeine into and through the skin over $24 \mathrm{~h}$, with no depletion of the donor material. As a percentage of the total applied drug dose, total caffeine delivery ranged from 6.6 and $7.4 \%$ for the aqueous solution and the conventional liposomes up to 14.9 and $16.0 \%$ for the transferosomes and niosomes, respectively. The greatest flux was seen for transferosomes and niosomes, with apparent flux increases between 12 and $24 \mathrm{~h}$ for these
28

Skin Pharmacol Physiol 2016;29:24-30 DOI: $10.1159 / 000441040$
Abd/Roberts/Grice 
formulations. Interestingly, these were the vesicles containing DPGluc as edge activators. This hydrophilic surfactant has also been shown to act as a penetration enhancer for dermal delivery of tretinoin when incorporated into liposomes [32], while other non-ionic surfactants caused similarly enhanced permeation fluxes, for example, with lidocaine [33]. A relatively constant flux after about $8 \mathrm{~h}$ was seen with the other formulations, including the liposomes containing eucalyptol and oleic acid. Given the relatively moderate enhancement of caffeine penetration seen with these latter vesicles, it seems likely that their actions as penetration enhancers in those formulations were not important factors. The combined functions of the surfactant edge activator and penetration enhancer would appear to explain the relative enhancement seen with DPGluc. While their work relates to the more lipophilic oestradiol, it is interesting to compare the finding of El Maghraby et al. [34], where greater penetration was seen with surfactant-containing liposomes than with oleic acid as a penetration enhancer alone. The authors suggested that liposomes increased oestradiol permeation by mechanisms that are additional to penetrationenhancing effects.

The niosomes and transferosomes also showed greater skin retention than other formulations. In particular, penetration into hair follicles from transferosomes appeared to be enhanced. The ultradeformable nature of these vesicles may allow them to gain access to these appendages more readily. Motwani and Rhein [35] suggested that polar drugs delivered from vehicles which are poorly miscible with sebum will have little chance to reach deeper parts of the hair follicles. On the other hand, lipophilic vehicles may assist the transport of drugs dissolved in the oil phase and into the pilosebaceous units [15]. Therefore, the enhancement of follicular penetration would rely on the solubility of the drug in the formulation and the compatibility of the formulation with the lipid environment of the sebum [35].

One way in which the non-ionic surfactant could enhance solute transfer from vesicles into the skin could be to assist in the absorption and fusion of the vesicle to the skin surface. This could maximize the ability of the vesicle to structurally modify the barrier properties of the skin, allowing the solute to pass more readily from its encapsulated environment into the intercellular regions of the stratum corneum. Studies [36-38] conclude the following: (1) the absorption and fusion of niosomes and transferosomes into the surface of skin enhance drug permeation, (2) the vesicles act as penetration enhancers to reduce the barrier properties of the stratum corneum and
(3) the lipid bilayers of niosomes act as a rate-limiting membrane barrier of solutes [39]. The possible mechanism for liposome and niosome formulations is the structural modification of the stratum corneum. For transferosomes, both phospholipid (DLPC) and non-ionic surfactant (DPGluc) can act as penetration enhancers, which are valuable for increasing the penetration of many drugs. These results suggest that the mechanism of drug transport across skin depends on the composition of the vesicle formulation and the solute used.

\section{Conclusion}

The in vitro permeation of caffeine from vesicles with different compositions of penetration enhancers and nonionic surfactant have been studied and evaluated. Incorporating the non-ionic surfactant DPGluc into liposomes enhanced the skin penetration of caffeine more than the penetration enhancers oleic acid and eucalyptol. The results suggest that the fusion of the vesicles with the lipid of the stratum corneum and the direct transfer of solute from the vesicle formulations to the skin may contribute to the mechanism of drug permeation enhancement by transferosomes and niosomes. The overall results indicate that the presence of penetration enhancers in the vesicle components is not the important operating factor.

\section{Acknowledgements}

This work was supported by the National Health and Medical Council of Australia (project grant APP1049906). We thank the Iraqi Ministry of Higher Education for a scholarship awarded to E.A.

\section{Disclosure Statement}

The authors have no conflicts of interest to declare.

\section{References}

Skin Pharmacol Physiol 2016;29:24-30 DOI: $10.1159 / 000441040$

\footnotetext{
1 Norlen L: Current understanding of skin barrier morphology. Skin Pharmacol Physiol 2013;26:213-216.

2 Wertz PW: Current understanding of skin biology pertinent to skin penetration: skin biochemistry. Skin Pharmacol Physiol 2013;26: 217-226.

3 Zhang Q, Grice JE, Li P, Jepps OG, Wang GJ, Roberts MS: Skin solubility determines maximum transepidermal flux for similar size molecules. Pharm Res 2009;26:1974-1985.
} 
4 Sharma VK, Sarwa KK, Mazumder B: Fluidity enhancement: a critical factor for performance of liposomal transdermal drug delivery system. J Liposome Res 2014;24:83-89.

5 Jacobs M, Martin GP, Marriott C: Effects of phosphatidylcholine on the topical bioavailability of corticosteroids assessed by the human skin blanching assay. J Pharm Pharmacol 1988;40:829-833.

6 Lademann J, Richter H, Meinke MC, LangeAsschenfeldt B, Antoniou C, Mak WC, Renneberg R, Sterry W, Patzelt A: Drug delivery with topically applied nanoparticles: science fiction or reality. Skin Pharmacol Physiol 2013;26:227-233

7 Williams AC: Brian Barry: Innovative contributions to transdermal and topical drug delivery. Skin Pharmacol Physiol 2013;26:234242.

8 Jain S, Jain P, Umamaheshwari R, Jain $\mathrm{N}$ : Transfersomes - a novel vesicular carrier for enhanced transdermal delivery: development, characterization, and performance evaluation. Drug Dev Ind Pharm 2003;29: 1013-1026.

9 Gupta A, Aggarwal G, Singla S, Arora R: Transfersomes: a novel vesicular carrier for enhanced transdermal delivery of sertraline: development, characterization, and performance evaluation. Sci Pharm 2012;80:10611080.

10 Cevc G, Gebauer D, Stieber J, Schätzlein A, Blume G: Ultraflexible vesicles, transferosomes, have an extremely low pore penetration resistance and transport therapeutic amounts of insulin across the intact mammalian skin. Biochim Biophys 1998;1368:201215.

11 Fang J-Y, Hong C-T, Chiu W-T, Wang Y-Y: Effect of liposomes and niosomes on skin permeation of enoxacin. Int J Pharm 2001;219: 61-72.

12 Cevc G, Schätzlein A, Blume G: Transdermal drug carriers: basic properties, optimization and transfer efficiency in the case of epicutaneously applied peptides. J Control Release 1995;36:3-16.

13 Paul A, Cevc G, Bachhawat B: Transdermal immunisation with an integral membrane component, gap junction protein, by means of ultradeformable drug carriers, transferosomes. Vaccine 1998;16:188-195.

14 Balakrishnan P, Shanmugam S, Lee WS, Lee WM, Kim JO, Oh DH, Kim D-D, Kim JS, Yoo BK, Choi H-G, Woo JS, Yong CS: Formulation and in vitro assessment of minoxidil niosomes for enhanced skin delivery. Int J Pharm 2009;377:1-8.
15 Manosroi A, Wongtrakul P, Manosroi J, Sakai $\mathrm{H}$, Sugawara F, Yuasa M, Abe M: Characterization of vesicles prepared with various nonionic surfactants mixed with cholesterol. Colloids Surf B Biointerfaces 2003;30:129-138.

16 Vora B, Khopade AJ, Jain NK: Proniosome based transdermal delivery of levonorgestrel for effective contraception. J Control Release 1998;54:149-165.

17 Manconi M, Sinico C, Valenti D, Lai F, Fadda AM: Niosomes as carriers for tretinoin. Iii. A study into the in vitro cutaneous delivery of vesicle-incorporated tretinoin. Int J Pharm 2006;311:11-19.

18 Hofland HEJ, van der Geest R, Bodde HE, Junginger HE, Bouwstra JA: Estradiol permeation from nonionic surfactant vesicles through human stratum corneum in vitro. Pharm Res 1994;11:659-664.

19 Blume-Peytavi U, Vogt A: Human hair follicle: reservoir function and selective targeting. Br J Dermatol 2011;165:13-17.

20 Grams YY, Bouwstra JA: Penetration and distribution of three lipophilic probes in vitro in human skin focusing on the hair follicle. J Control Release 2002;83:253-262.

21 Lademann J, Knorr F, Richter H, Blume-Peytavi U, Vogt A, Antoniou C, Sterry W, Patzelt A: Hair follicles - an efficient storage and penetration pathway for topically applied substances. Skin Pharmacol Physiol 2008;21: 150-155.

22 Herkenne C, Alberti I, Naik A, Kalia YN, Mathy F-X, Préat V, Guy RH: In vivo methods for the assessment of topical drug bioavailability. Pharm Res 2008;25:87-103.

23 Teichmann A, Jacobi U, Ossadnik M, Richter H, Koch S, Sterry W, Lademann J: Differential stripping: determination of the amount of topically applied substances penetrated into the hair follicles. J Gen Intern Med 2005;20: 264-269.

24 Geusens B, Van Gele M, Braat S, De Smedt SC, Stuart MCA, Prow TW, Sanchez W, Roberts MS, Sanders NN, Lambert J: Flexible nanosomes (SECosomes) enable efficient siRNA delivery in cultured primary skin cells and in the viable epidermis of ex vivo human skin. Adv Funct Mater 2010;20:4077-4090.

25 Verma DD, Fahr A: Synergistic penetration enhancement effect of ethanol and phospholipids on the topical delivery of cyclosporin A. J Control Release 2004;97:55-66.
26 Budai L, Kaszas N, Grof P, Lenti K, Maghami K, Antal I, Klebovich I, Petrikovics I, Budai M: Liposomes for topical use: a physico-chemical comparison of vesicles prepared from egg or soy lecithin. Sci Pharm 2013;81:1151-1166.

27 Fadda AM, Baroli BM, Maccioni AM, Sinico C, Valenti D, Alhaique F: Phospholipid-detergent systems: effects of polysorbates on the release of liposomal caffeine. Il. Farmaco 1998;53:650-654.

28 Ntimenou V, Fahr A, Antimisiaris SG: Elastic vesicles for transdermal drug delivery of hydrophilic drugs: a comparison of important physicochemical characteristics of different vesicle types. J Biomed Nanotechnol 2012;8: 613-623.

29 El Maghraby GM, Williams AC, Barry BW: Can drug-bearing liposomes penetrate intact skin? J Pharm Pharmacol 2006;58:415-429.

30 El Maghraby GM, Barry BW, Williams AC: Liposomes and skin: from drug delivery to model membranes. Eur J Pharm Sci 2008;34: 203-222.

31 Williams AC, Barry BW: Skin absorption enhancers. Crit Rev Ther Drug Carrier Syst 1992;9:305-353.

32 Manconi M, Sinico C, Caddeo C, Vila AO, Valenti D, Fadda AM: Penetration enhancer containing vesicles as carriers for dermal delivery of tretinoin. Int J Pharm 2011;412:3746.

33 Sarpotdar PP, Zatz JL: Evaluation of penetration enhancement of lidocaine by nonionic surfactants through hairless mouse skin in vitro. J Pharm Sci 1986;75:176-181.

34 El Maghraby GM, Williams AC, Barry BW: Skin delivery of oestradiol from lipid vesicles: importance of liposome structure. Int J Pharm 2000;204:159-169.

35 Motwani MR, Rhein LD: Influence of vehicles on the phase transitions of. J Cosmet Sci 2002 53:35-42.

36 El Maghraby GM, Williams AC, Barry BW: Skin delivery of 5-fluorouracil from ultradeformable and standard liposomes in-vitro. J Pharm Pharmacol 2001;53:1069-1077.

37 Schreier H, Bouwstra J: Liposomes and niosomes as topical drug carriers: dermal and transdermal drug delivery. J Control Release 1994;30:1-15.

38 Uchegbu IF, Vyas SP: Non-ionic surfactant based vesicles (niosomes) in drug delivery. Int J Pharm 1998;172:33-70.

39 Alsarra IA, Bosela AA, Ahmed SM, Mahrous GM: Proniosomes as a drug carrier for transdermal delivery of ketorolac. Eur J Pharm Biopharm 2005;59:485-490. 